\title{
Hematocrit, As Part of an Integrated Condition Assessment System of the Body
}

\author{
Andrey Belousov* \\ Laboratory Applied Nanotechnology of Belousov, Department of Anesthesiology, Intensive Care, \\ Transfusiology, Hematology, Kharkov Medical Academy of Postgraduate Education, Ukraine
}

\begin{abstract}
*Corresponding Author: Andrey Belousov, Laboratory Applied Nanotechnology of Belousov, Department of Anesthesiology, Intensive Care, Transfusiology, Hematology, Kharkov Medical Academy of Postgraduate Education, Ukraine, Email: an.belousov2012@ukr.net
\end{abstract}

\begin{abstract}
The focus of the article is rather situated on current faults and recommendations for transfusion of red blood assessment, clinical evaluation of changes in hematocrit. The main task of therapy for acute massive blood loss is not urgent thoughtless transfusion of red blood cells for the fast recovery of the hemoglobin and hematocrit levels. The oxygen-carrying capacity of blood does not directly reflect the delivery of oxygen to tissues. The severity of the patient's condition depends of individual ability of the organism to resist hypoxia, mechanisms resulting in physiological compensation for the anemia caused by blood loss. The main tasks of therapy are timely maintaining appropriate and effective compensatoryadaptive reactions of an organism, providing of the sanogenetic processes. Quickly and comfortable algorithm assessment changes in hematocrit was presented for used in practice. Objective analysis hematocrit and hemoglobin levels should be carried out only in combination with data on blood pressure, pulse rate, respiratory rate, urine output and shock index.
\end{abstract}

Keywords: hematocrit, hemoglobin, assessment system, blood loss, transfusion of red blood cells.

\section{INTRODUCTION}

In modern health care today, blood transfusion plays a vital role. Blood transfusion can alleviate health and save life if used appropriately. According to WHO [1], appropriate use of blood products is defined as "the transfusion of safe blood products only to treat a condition leading to significant morbidity or mortality that cannot be prevented or managed effectively by other means".

The accumulation of fundamental knowledge in the field of Hematology, Immunohematology, Pathophysiology, Biochemistry, Biophysics, Histology and other related medical specialties objectively contributed to the formation of a new medical discipline - Clinical Transfusiology. Unfortunately, today among some anesthesiologists and resuscitators there is an erroneous and potentially dangerous point of view regarding the importance of this discipline. Inertia of traditional views on blood loss, and an archaism of fundamental knowledge in the field of Clinical Transfusiology negatively affect the quality of writing clinical protocols of blood transfusion. The presence of many incorrect scientific articles in various peer-reviewed surgical journals (including journals with a high citation rating) forms a false idea among doctors about the pathophysiological mechanisms of blood loss, an adequate assessment of severity of the clinical condition of the patient. Such publications deprive the understanding of the mechanisms of compensatory systems of the organism, which resist massive blood loss and protect the body from death. Intensive care physicians and anesthesiologists should be aware that from the point of view of pathophysiologically, to maintain the compensatory reactions of the organism, primarily, the therapy must be directed at the elimination an acute deficit in circulating blood volume (CBV), secondly, the correction of coagulation factors, and only in the last instance - to a possible increase in the number of the oxygen-carrier [2].The severity of the patient's condition in acute massive blood loss does not depend directly on the indicators hematocrit and hemoglobin. The severity of the patient's condition determines the individual ability of the organism to resist hypoxia, the effectiveness of compensatory systems [3]. Therefore, the development and improvement of personalized express-diagnostic systems of functional state of 
the organism and, estimation algorithms of the patient's severity are some of the main vectors of the objective approach to solve the issue of timeliness and effectiveness the prescribing transfusion of red blood cells. For example, today one of the alternative variants of such evaluation system can be the author's universal analytical system of physiological condition of the body (PHUAS) [4]. The main task of therapy for acute massive blood loss is not urgent thoughtless application of blood transfusion for the fast recovery of the levels of hematocrit and hemoglobin, and the timely establishment of adequate conditions for maintaining functioning of compensatory systems of the organism and providing of the sanogenetic processes.

The main purpose of this article is to teach of physicians to interpret correctly the different of variants of the hematocrit changes as part of an integrated system of assessing the state of the organism.

Table1. The variants of changes in hematocrit on the background of the basic organism physiological parameters.

\begin{tabular}{|l|l|l|l|l|l|l|}
\hline Variants & HCT & \multicolumn{1}{c|}{ Pulse rate } & \multicolumn{1}{c|}{ BP } & Urine output & \multicolumn{1}{c|}{ Respiration rate } & Shock index \\
\hline I & $\downarrow$ & $\uparrow$ & Normal & Normal & Normal/ $\uparrow$ Underload & $\uparrow$ \\
\hline II & $\downarrow$ & Normal/ $\downarrow$ & $\uparrow$ & Normal $/ \downarrow$ & Normal/ $\uparrow$ & $\downarrow$ \\
\hline III & $\downarrow$ & $\uparrow \uparrow$ & $\downarrow \downarrow$ & $\downarrow \downarrow /$ Anuria & $\uparrow \uparrow$ & $\uparrow \uparrow \uparrow$ \\
\hline IV & $\uparrow$ & Normal $/ \uparrow$ & Normal $/ \uparrow$ & $\uparrow / \downarrow$ & Normal $/ \uparrow$ & Normal $/ \uparrow$ \\
\hline
\end{tabular}

Notes: HCT-hematocrit; BP - blood pressure; Shock index = Pulse rate/Systolic bloodpressure $\left(\right.$ normal=-0.2 $\left.2^{n}\right)$

\section{DIS CUSSION}

Variant I: The level of hematocrit decreased against the background of moderate tachycardia and tachypnea, which increase after physical activity. Blood pressure and hourly urine output are normal. The shock index is moderately elevated. This variant of hematocrit reduction characterizes hemic hypoxia and in the absence of dynamics of hemoglobin reduction an transfusion of red blood cells is not advisable.

Variant II: The level of hematocrit decreased against the background of normal or slight decrease in heart rate. Blood pressure is elevated. Urine output is reduced or normal. The shock index is always below normal $(<0.54)$. This variant characterizes the state of hypervolemia. At the same time, the implementation of transfusion of red blood cells in order to increase the level of hematocrit is not only unjustified, but also dangerous for the patient's life due to the volume overload of the small circle blood circulation.

Variant III: The level of hematocrit decreased against the background of severe tachycardia and tachypnea, reduced blood pressure and hourly diuresis, to the manifestation of anuria. Elevated shock index $(>1.5)$. This variant to reduce the level of hematocrit is in a mixed form of hypoxia (circulatory + hemic hypoxia), which is characteristic of massive blood loss.

Variant IV: The level of hematocrit increased against the background of normal or increased pulse rate, blood pressure and diuresis. At the same time, the dynamics of hematocrit increase may be accompanied by a decrease in hourly diuresis. Shock index is up. This variant of hematocrit level change is typical for hypovolemic polycythemia.

\section{CONClusion}

Thus, hematocrit and hemoglobin levels not only do not objectively reflect degree of blood loss and patient's severity but also a priori are not targeted indicators for transfusion of red blood cells. Objective analysis hematocrit and hemoglobin levels should be carried out only in combination with data on blood pressure, pulse rate, respiratory rate, urine output and shock index. Recommendations for transfusion of red blood cells which are based only on hematocrit and hemoglobin data are not justified and unsafe for the patient.

\section{REFERENCES}

[1] World Health Organization. The clinical use of blood in obstetrics, pediatrics. Surgery\& Anaesthesia, Trauma \& Burns; 2001.

[2] Belousov A., Malygon E, Yavorskiy V. Calculating the True Volume of Blood Loss Journal of Anesthesia \& Clinical Research. Volume 8. Issue 11. 2017. ISSN: 2155-6148 DOI:10.4172/2155-6148.1000787

[3] Pope A, French G, Longnecker DE, editors. Fluid Resuscitation: State of the Science for Treating Combat Casualties and Civilian Injuries. Institute of Medicine (US) Committee on Fluid Resuscitation for Combat Casualties. Washington (DC): National Academies Press (US); 1999. Bookshelf ID: NBK224596PMID: 25077227DOI: $10.17226 / 9625$ 
Hematocrit, As Part of an Integrated Condition Assessment System of the Body

[4] Belousov A. New Program for Estimation of Degree of the Patient's Severity. Journal of Anesthesia and Surgery. Ommega Publisher.
NJ-USA，2015，2(2): 1-3. DOI: 10.15436/23771364.15.007

Citation: Andrey Belousov. Hematocrit, As Part of an Integrated Condition Assessment System of the Body. ARC Journal of Anesthesiology. 2018;3(1):15-17. DOI: dx.doi.org/10.20431/2455-9792.0301004.

Copyright: (C) 2018 Authors. This is an open-access article distributed under the terms of the Creative Commons Attribution License, which permits unrestricted use, distribution, and reproduction in any medium, provided the original author and source are credited. 